\title{
Blowup of solutions for nonlinear nonlocal heat equations
}

\author{
Piotr Biler $^{1}$ (D)
}

Received: 11 July 2018 / Accepted: 27 January 2019 / Published online: 4 February 2019

(c) The Author(s) 2019

\section{Abstract}

Blowup analysis for solutions of a general evolution equation with nonlocal diffusion and localized source is performed. Sufficient conditions for blowup are expressed in terms of some Morrey space norms. A comparison of these with recent results on global-in-time solutions is discussed.

Keywords Nonlinear nonlocal heat equation - Blowup of solutions · Comparison of size of global/blowing up solution

Mathematics Subject Classification 35K55 · 35B44

\section{Introduction}

We consider here nonnegative solutions $u=u(x, t) \geq 0$ of the Cauchy problem

$$
\begin{aligned}
& u_{t}=\mathcal{A} u+F(u), \quad x \in \mathbb{R}^{d}, t>0, \\
& u(x, 0)=u_{0}(x) \geq 0, \quad x \in \mathbb{R}^{d},
\end{aligned}
$$

with the linear nonlocal diffusion operator

$$
\mathcal{A} u(x)=(J * u)(x)-u(x)
$$

defined by the convolution of $u$ with a nonnegative radially symmetric function $J$ satisfying $\int_{\mathbb{R}^{d}} J(x) \mathrm{d} x=1$, and with the nonlinearity (a localized source) defined by

Communicated by A. Constantin.

The author, partially supported by the NCN Grant 2016/23/B/ST1/00434, thanks Philippe Souplet and Mikołaj Sierżęga for many interesting conversations and the referees for helpful comments.

Piotr Biler

Piotr.Biler@math.uni.wroc.pl

1 Instytut Matematyczny, Uniwersytet Wrocławski, pl. Grunwaldzki 2/4, 50-384 Wrocław, Poland 
a locally Lipschitz convex function $F:[0, \infty) \rightarrow[0, \infty), F(0)=0$, satisfying the condition

$$
\int^{\infty} \frac{\mathrm{d} u}{F(u)}<\infty .
$$

Similar evolution equations extending the classical nonlinear heat equation thoroughly presented in [17]

$$
u_{t}=\Delta u+|u|^{p-1} u, \quad x \in \mathbb{R}^{d}, \quad t>0,
$$

and the equations with nonlocal diffusion operators defined by fractional powers of the Laplacian, $\alpha \in(0,2)$

$$
u_{t}=-(-\Delta)^{\alpha / 2} u+|u|^{p-1} u, \quad x \in \mathbb{R}^{d}, \quad t>0,
$$

and even more general nonlinearities, have been studied in, e.g., [11] (the linear case), and e.g., $[1,13,20]$ (the nonlinear case).

Equations of the type (1) are related to the differential and integrodifferential equations (5) and (6) by their long time asymptotic behavior determined frequently by the linear equations (12)-(13) below, and studied in, e.g., [1,11,13].

There are plenty of results on closely related questions on conditions on the initial data guaranteeing the local-in-time existence of solutions to Eq. (5) and sufficient conditions leading to finite time blowup of solutions, see $[2,3,14]$, the latter reference dealing with a general nondecreasing but not necessarily convex nonlinearity.

A standard theory of the existence of solutions for problem (1)-(2) in $[1,11,13]$ is developed in the framework of mild solutions, i.e., those satisfying the Duhamel formula

$$
u(t)=\mathrm{e}^{t \mathcal{A}} u_{0}+\int_{0}^{t} \mathrm{e}^{(t-s) \mathcal{A}} F(u(s)) \mathrm{d} s .
$$

Here, $u \in \mathcal{C}\left([0, T), L^{1}\left(\mathbb{R}^{d}\right)\right)$ is required when $F \equiv 0$, i.e., in the case of linear equations (1). Note that the semigroup $\mathrm{e}^{t \mathcal{A}}$ is strongly continuous, so that the initial condition $u_{0}$ is attained in the sense of $L^{1}$-limit as $t \rightarrow 0$. In the case of nonlinear equations with, say, $|F(u)| \leq C\left(1+|u|^{p}\right)$, assumption $u_{0} \in L^{1} \cap L^{\infty}\left(\mathbb{R}^{d}\right)$ guarantees the local-in-time well-posedness of the Cauchy problem (1)-(2) with

$$
u \in \mathcal{C}^{1}\left((0, T), L^{1}\left(\mathbb{R}^{d}\right) \cap L^{\infty}\left(\mathbb{R}^{d}\right)\right) \cap \mathcal{C}\left([0, T), L^{1}\left(\mathbb{R}^{d}\right) \cap L^{\infty}\left(\mathbb{R}^{d}\right)\right),
$$

see $[11,13]$. In both the cases mild solutions are weak solutions so that, in particular,

$$
\begin{gathered}
\int\left(u(x, t) \phi(x, t)-u_{0}(x) \phi(x, 0)\right) \mathrm{d} x-\int_{0}^{t} \int u(x, s) \frac{\partial}{\partial t} \phi(x, s) \mathrm{d} x \mathrm{~d} s \\
=\int_{0}^{t} \int u(x, s) \mathcal{A} \phi(x, s) \mathrm{d} x \mathrm{~d} s+\int_{0}^{t} \int F(u(x, s)) \phi(x, s) \mathrm{d} x \mathrm{~d} s
\end{gathered}
$$

holds for $t<T$ and each function $\phi \in \mathcal{C}^{2,1}\left(\mathbb{R}^{d} \times[0, T)\right) \cap L^{\infty}\left(\mathbb{R}^{d} \times(0, T)\right)$, which is completely analogous to the concept of weak solutions of nonlinear heat equations with either the Laplacian (5) or fractional Laplacian (6). 
However, it is convenient to adopt here a slightly more general definition of locally bounded solutions

$$
u \in \mathcal{C}^{1}\left((0, T), L^{1}\left(\mathbb{R}^{d}\right) \cap L_{\mathrm{loc}}^{\infty}\left(\mathbb{R}^{d}\right)\right) \cap \mathcal{C}\left([0, T), L^{1}\left(\mathbb{R}^{d}\right) \cap L_{\mathrm{loc}}^{\infty}\left(\mathbb{R}^{d}\right)\right),
$$

admitting unbounded initial data which are merely in $L^{1} \cap L_{\mathrm{loc}}^{\infty}\left(\mathbb{R}^{d}\right)$. This is motivated by the fact that solutions to problem (1)-(2) with data in $L^{1} \cap L_{\text {loc }}^{q}\left(\mathbb{R}^{d}\right)$ for some particular $q$ may exist, see [14] in the case of equations like (5), (6) with general nonlinearities. Note that this definition permits to consider locally bounded in space but unbounded solutions as in [4,12].

Remark that unlike the case of the Eqs. (5) and (6), we cannot expect that solutions are smooth for $t>0$, i.e., an instantaneous regularization effect of the semigroup $\mathrm{e}^{t \mathcal{A}}$ generated by bounded diffusion operators $\mathcal{A}$ on solutions is absent.

The phenomenon of a blowup of a solution is understood here in the local $L^{\infty}$ sense, i.e., $u$ is a blowing up solution not later than at $t=T>0$ if for some $R>0$ the relation

$$
\limsup _{t \nearrow T} \sup _{|x|<R} u(x, t)=\infty
$$

holds. This definition is consistent with the above meaning of solutions requiring $u(t) \in L^{1}\left(\mathbb{R}^{d}\right) \cap L_{\mathrm{loc}}^{\infty}\left(\mathbb{R}^{d}\right)$ for $t>0$.

Theorem 1 on local-in-time solutions to Eq. (1) that cannot be continued to globalin-time ones is one of the main results in this paper. Proposition 3 interprets a general condition in Theorem 1 in terms of the Morrey space norms related to approximative scaling properties of the problem. There are also subsidiary results on the size of global-in-time solutions compared to blowing up ones in the case of Eqs. (5) and (6).

The main idea here is that we are looking for a single quantity $\ell=\ell\left(u_{0}\right)$ (a functional norm) which decides on the blowup versus global existence. Unfortunately, we do not have a dichotomic partition of the set of admissible initial data but weaker results like: $\ell\left(u_{0}\right)<c$ implies the global existence while $\ell\left(u_{0}\right)>C$ (with $C>c$ ) leads to a blowup of solutions in Theorem 5. Quite often the condition $\ell\left(u_{0}\right)<\infty$ is necessary, but not always sufficient, for the local-in-time existence of solutions, see $[4,18]$ for the cases of Eqs. (5) and (6).

Notation The homogeneous Morrey spaces $M_{q}^{s}\left(\mathbb{R}^{d}\right)$ modeled on the Lebesgue space $L^{q}\left(\mathbb{R}^{d}\right), q \geq 1$, are defined for $u \in L_{\mathrm{loc}}^{q}\left(\mathbb{R}^{d}\right)$ and $1 \leq q \leq s<\infty$, by their norms

$$
\begin{aligned}
|u|_{M_{q}^{s}} & \equiv\left(\sup _{R>0, x \in \mathbb{R}^{d}} R^{d(q / s-1)} \int_{B(x, R)}|u(y)|^{q} \mathrm{~d} y\right)^{1 / q} \\
& =\sup _{R>0, x \in \mathbb{R}^{d}} R^{d(1 / s-1 / q)}\left\|\mathbf{I}_{B(x, R)} u\right\|_{q}<\infty
\end{aligned}
$$


with the convention $M_{1}^{s}\left(\mathbb{R}^{d}\right)=M^{s}\left(\mathbb{R}^{d}\right)$. Here, $B(x, R)$ denotes the ball centred at $x$ of radius $R:\{y:|y-x|<R\}$, and $\mathbf{I}_{B(x, R)}$ is its characteristic function.

The asymptotic relation $f \approx g$ as $s \rightarrow s_{0}$ (with either $s_{0}=0$ or $s_{0}=\infty$ ) means that $\lim _{s \rightarrow s_{0}} \frac{f(s)}{g(s)}=1$, and $f \asymp g$ is used whenever $\lim _{s \rightarrow s_{0}} \frac{f(s)}{g(s)} \in(0, \infty)$. We will use the notation $g \gg 1$ if the quantity $g$ (depending on some parameters) is supposed to be sufficiently large (in terms of those parameters).

\section{Blowup for a general nonlinear source, nonlocal diffusion model}

Our aim in this paper is to give a simple proof of blowup of solutions for the Cauchy problem (1)-(2) based on the classical idea of Fujita [12]. We believe that this proof is simpler than monotonicity arguments given in [1]. Moreover, this argument applies to a class of initial data much larger than in [1, Theorem 2.4], giving explicit and rather precise general sufficient conditions on functions $u_{0}$ in (2) in order to solutions of (1)-(2) blow up in a finite time, together with estimates of the blowup time, cf. also [20] for Eq. (6).

Let us briefly recall some facts from Alfaro [1] and Chasseigne et al. [11]. The linear nonlocal diffusion operator $\mathcal{A}: L^{1}\left(\mathbb{R}^{d}\right) \rightarrow L^{1}\left(\mathbb{R}^{d}\right)$ defined in (3) is bounded and generates the semigroup of linear operators $\mathrm{e}^{t \mathcal{A}}$. This semigroup is represented as convolutions with kernels $k_{t} \in L^{1}\left(\mathbb{R}^{d}\right)$, normalized so that $\int_{\mathbb{R}^{d}} k_{t}(x) \mathrm{d} x=1$, defined by the inverse Fourier transform $\mathcal{F}^{-1}$ on $\mathbb{R}^{d}$

$$
k_{t}(x)=\mathcal{F}^{-1}\left(\mathrm{e}^{t(\widehat{J}(\xi)-1)}\right)(x)
$$

Typical and the most interesting examples of functions $J$ are those with their Fourier transforms $\widehat{J}$ satisfying

$$
\widehat{J}(\xi)-1 \approx-A|\xi|^{\alpha}, \quad \text { as } \quad \xi \rightarrow 0
$$

with $A>0, \alpha=2$ (corresponding to, e.g., the case of smooth, compactly supported functions $J)$, and those with $\alpha \in(0,2)$, cf. [11, Sec. 1]. Note that for $\alpha \in(0,2)$ such $J$ do not have finite second moment. As it was studied in [11], the long time asymptotics of solutions of the linear Cauchy problem

$$
v_{t}=\mathcal{A} v, \quad v(., 0)=v_{0}
$$

is then determined either by that of the classical heat equation

$$
z_{t}=\Delta z \text { if } \quad \alpha=2
$$

or the fractional heat equation

$$
z_{t}=-(-\Delta)^{\alpha / 2} z \text { in the case } \alpha \in(0,2)
$$


see (22) below for a precise statement from Chasseigne et al. [11, Theorem 1]. Other examples of functions $J$ with the Fourier transform $\widehat{J}$ like $\widehat{J}(\xi)-1 \approx A|\xi|^{2} \log |\xi|$ as $|\xi| \rightarrow 0$, are mentioned in [11, Th. 5.1], and then $J(x) \asymp \frac{1}{|x|^{d+2}}$ as $|x| \rightarrow \infty$. Thus, they are examples of operators $\mathcal{A}$ whose kernels have "heavy tails": $J(x) \asymp \frac{1}{|x|^{n}}$ as $|x| \rightarrow \infty$ with some $n>d$, discussed in [1,11]. For them, if $n \in(d, d+2)$, then $\alpha=n-d \in(0,2)$ holds. Their semigroup kernels have also heavy tails unlike the Gauss-Weierstrass kernel of the heat semigroup for $\alpha=2$. Besides bounded diffusion operators (3) studied here, the proof of Theorem 1 below applies also to unbounded operators $\Delta$ and $-(-\Delta)^{\alpha / 2}$ as was in classical papers $[12,20]$.

Theorem 1 Define the function $h(w)=\int_{w}^{\infty} \frac{\mathrm{d} u}{F(u)}$. Suppose that $u_{0} \geq 0$ satisfies for some $T>0$ the condition

$$
\frac{W_{T}(0)}{h^{-1}(T)}>1
$$

with the moment $W_{T}(t)$ defined for $t \in[0, T)$ with the kernels $k_{t}$ in (10) as

$$
W_{T}(t)=\int_{\mathbb{R}^{d}} k_{T-t}(x) u(x, t) \mathrm{d} x .
$$

Then, any local in time classical solution $u=u(x, t)$ of the Cauchy problem (1)-(2) cannot be continued beyond $t=T$.

Proof The function $h$ is decreasing and satisfies $h(0)=\infty, h(\infty)=0$, by assumption (4) on the convex function $F$. By definition (15), we have $W_{T}(t)=k_{T-t} * u(., t)(0)$ and, of course, $z(., t)=k_{T-t}$ solves the backward diffusion equation with the terminal condition

$$
z_{t}=-\mathcal{A} z, \quad z(., T)=\delta_{0} .
$$

Following the idea in [12], let us compute the time derivative of $W_{T}(t)$

$$
\begin{aligned}
\frac{\mathrm{d}}{\mathrm{d} t} W_{T}(t)= & \int k_{T-t}(x) \frac{\partial}{\partial t} u(x, t) \mathrm{d} x+\int \frac{\partial}{\partial t} k_{T-t}(x) u(x, t) \mathrm{d} x \\
= & \int k_{T-t}(x)(\mathcal{A} u)(x, t) \mathrm{d} x+\int k_{T-t}(x) F(u(x, t)) \mathrm{d} x \\
& +\int\left(-\mathcal{A} k_{T-t}(x)\right) u(x, t) \mathrm{d} x \\
= & \int k_{T-t}(x) F(u(x, t)) \mathrm{d} x \\
\geq & F\left(W_{T}(t)\right)
\end{aligned}
$$

by the symmetry property of the function $J$, so that the symmetry of the semigroup, and the Jensen inequality in the last line. Integrating this from 0 to $t$, we obtain

$$
h\left(W_{T}(0)\right)-h\left(W_{T}(t)\right) \geq t .
$$


If initially $W_{T}(0)>h^{-1}(T)$ holds, then taking into account the property $\lim _{w \searrow 0} h(w)=\infty$, we arrive at

$$
\lim _{t \nearrow T} W_{T}(t)=\infty
$$

Finally, if $\lim _{t \nearrow T} W_{T}(t)=\infty$ then evidently $\lim \sup _{t \nearrow T} \sup _{|x|<R} u(x, t)=\infty$ for every $R>0$ which means that $u$ blows up not later than at $t=T$. Indeed, we have $\lim _{t \nearrow T} \sup _{|x|>R} k_{T-t}(x)=0$ for every $R>0$.

Remark 2 In some particular cases, under more restrictive assumptions than condition (4), the sufficient condition for blowup of solutions of (1)-(2) can be described in a more explicit way than condition

$$
\sup _{T>0} \frac{W_{T}(0)}{h^{-1}(T)}>1
$$

considered in Theorem 1.

For instance, for the nonlinear heat equation (5) with $\mathcal{A} u=\Delta u$ and $F(u)=u^{p}$, so that for $\mathrm{e}^{t \Delta}$ defined with the Gauss-Weierstrass kernel, the condition (17) is equivalent to

$$
\sup _{T>0} T^{\frac{1}{p-1}}\left\|\mathrm{e}^{T \Delta} u_{0}\right\|_{\infty}>\left(\frac{1}{p-1}\right)^{\frac{1}{p-1}} .
$$

This is a sufficient condition for the blowup of (5) derived in [12], and this has been interpreted in a recent paper [4] in terms of the Morrey space norm $M^{d(p-1) / 2}\left(\mathbb{R}^{d}\right)$.

We have, in this direction, the following general

Proposition 3 (i) If the infinitesimal generator $\mathcal{A}$ of the semigroup $\mathrm{e}^{t \mathcal{A}}$ satisfies (11) for an $\alpha \in(0,2], F(u) \asymp u^{p}$ with some $p>1$ as $s \rightarrow 0, u_{0} \in L^{1}\left(\mathbb{R}^{d}\right), \widehat{u_{0}} \in L^{1}\left(\mathbb{R}^{d}\right)$, then

$$
\sup _{T>0} T^{\frac{1}{p-1}}\left\|\mathrm{e}^{-T(-\Delta)^{\alpha / 2}} u_{0}\right\|_{\infty} \gg 1
$$

is a sufficient condition of blowup of solution of problem (1)-(2). Condition (19) is equivalent to a large value of the Morrey space norm of $u_{0}$

$$
\left\|u_{0}\right\|_{M^{d(p-1) / \alpha}} \gg 1
$$

(ii) Moreover, if $1<p<p_{\mathrm{F}}$ where $p_{\mathrm{F}}=1+\frac{\alpha}{d}$ is the so-called Fujita exponent, then each nontrivial nonnegative solution $u \not \equiv 0$ blows up in a finite time.

Proof (i) Clearly, for $p>1$, if $F(u) \asymp u^{p}$, that is $F(u) \approx c u^{p}$ with some $c>0$ as $u \searrow 0$, so $h(u) \approx \frac{1}{c(p-1)} u^{1-p}, u \searrow 0$. Thus, $h^{-1}(z) \approx z^{-\frac{1}{p-1}}\left(\frac{1}{c(p-1)}\right)^{\frac{1}{p-1}}$ as $z \rightarrow \infty$. Therefore, the sufficient condition for blowup (17) becomes

$$
\exists T>0: \quad T^{\frac{1}{p-1}}\left|\mathrm{e}^{T \mathcal{A}} u_{0}(0)\right| \gg 1 .
$$


By the translational invariance of Eq. (1), condition (21) for $u_{0} \geq 0$ is equivalent to

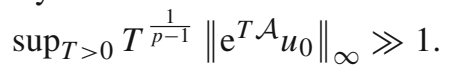

On the other hand, according to [11, Theorem 1], for $u_{0} \in L^{1}\left(\mathbb{R}^{d}\right)$ with $\widehat{u_{0}} \in$ $L^{1}\left(\mathbb{R}^{d}\right)$ and large $t>0$, the semigroup $\mathrm{e}^{t \mathcal{A}}$ applied to $u_{0}$ can be well approximated by the semigroup $\mathrm{e}^{-t(-\Delta)^{\alpha / 2}}$ generated by the fractional power of the Laplacian $-(-\Delta)^{\alpha / 2}$

$$
\lim _{t \rightarrow \infty} t^{\frac{d}{\alpha}}\left\|\mathrm{e}^{t \mathcal{A}} u_{0}-\mathrm{e}^{-t(-\Delta)^{\alpha / 2}} u_{0}\right\|_{\infty}=0
$$

while $\left\|\mathrm{e}^{-t(-\Delta)^{\alpha / 2}} u_{0}\right\|_{\infty}=\mathcal{O}\left(t^{-\frac{d}{\alpha}}\right)$.

Next, we have the equivalence

$$
\sup _{t>0} t^{\gamma}\left\|\mathrm{e}^{-t(-\Delta)^{\alpha / 2}} u\right\|_{\infty}<\infty \text { if and only if } u \in B_{\infty, \infty}^{-\gamma \alpha}\left(\mathbb{R}^{d}\right)
$$

where $B_{\infty, \infty}^{-\kappa}$ is the homogeneous Besov space of order $-\kappa<0$. The above condition (23) is for $u \geq 0$ equivalent to $u \in M^{\frac{d}{\alpha \gamma}}\left(\mathbb{R}^{d}\right)$, the Morrey space of order $\frac{d}{\alpha \gamma}$. For these issues, see the discussion in [15, Prop. 2B)] for $\alpha=2$ and the argument in [16, Sec. 4, p. 667, proof of Prop. 2] for $\alpha \in(0,2)$.

Finally, condition (21) is equivalent to

$$
\exists T>0: \quad T^{\frac{1}{p-1}}\left|\mathrm{e}^{-T(-\Delta)^{\alpha / 2}} u_{0}(0)\right| \gg 1
$$

which is, in turn, equivalent for $u_{0} \geq 0$ to condition (20) by the above remarks.

Note that for $\mathrm{e}^{t \mathcal{A}}=\mathrm{e}^{-t(-\Delta)^{\alpha / 2}}$ the assumptions on the initial data $u_{0} \in L^{1}\left(\mathbb{R}^{d}\right)$ with $\widehat{u_{0}} \in L^{1}\left(\mathbb{R}^{d}\right)$ can be relaxed to $u_{0} \in L^{1}\left(\mathbb{R}^{d}\right) \cap L^{\infty}\left(\mathbb{R}^{d}\right)$.

Of course, condition (24) is quite general, and involves one free parameter $T>0$. Particular examples of initial data considered in [1, Th. 2.3] leading to blowup of solutions do satisfy (24).

(ii) Rewriting the quantity in (24) as

$$
\begin{aligned}
T^{\frac{1}{p-1}}\left|\mathrm{e}^{-T(-\Delta)^{\alpha / 2}} u_{0}(0)\right| & =T^{\frac{1}{p-1}-\frac{d}{\alpha}} \int R\left(\frac{|x|}{T^{\frac{1}{\alpha}}}\right) u_{0}(x) \mathrm{d} x \\
& \approx R(0) T^{\frac{1}{p-1}-\frac{d}{\alpha}}\left\|u_{0}\right\|_{1} \rightarrow \infty, \text { for } T \rightarrow \infty
\end{aligned}
$$

we see that for each $p<1+\frac{\alpha}{d}$ and $\left\|u_{0}\right\|_{1}>0$, the upper bound equals $\infty$ as claimed; remember relation (22). Above, the kernel of the semigroup $\mathrm{e}^{-t(-\Delta)^{\alpha / 2}}$ has the selfsimilar form $P_{t, \alpha}(x, t)=t^{-\frac{d}{\alpha}} R\left(\frac{|x|}{T^{\frac{1}{\alpha}}}\right)$ with a smooth function $R$. This kernel satisfies the bound

$$
0<P_{t, \alpha}(x) \leq \frac{C}{\left(t^{1 / \alpha}+|x|\right)^{d}}
$$

see for instance $[11,16]$. 
The proof of (ii) for $p \leq p_{\mathrm{F}}$ and $\mathrm{e}^{t \mathcal{A}}=\mathrm{e}^{-t(-\Delta)^{\alpha / 2}}, \alpha \in(0,2)$, is in [20]. A rather short new proof of the result (ii) for $\alpha=2$ and $p=p_{\mathrm{F}}$ is in [4].

These are counterparts of results in [4, Remark 7, Theorem 2] for the classical nonlinear heat equation. These, together with results of [8, Proposition 2.3], lead to the following result, similarly as was in [4, Corollary 11] for the Cauchy problem (6) with (2). For analogous questions for radial solutions of chemotaxis systems, see also [5].

Corollary 4 There exist two positive constants $c(\alpha, d, p)$ and $C(\alpha, d, p)$ such that if $p>1+\frac{\alpha}{d}$ then

(i) $\left|u_{0}\right|_{M_{q}^{d(p-1) / \alpha}}<c(\alpha, d, p)$ for some $q \in\left(1, \frac{d(p-1)}{\alpha}\right)$, implies that problem (6) with data (2) has a global in time, smooth solution which, moreover, satisfies the time decay estimate $\|u(t)\|_{\infty}=\mathcal{O}\left(t^{-1 /(p-1)}\right)$.

(ii) $\left|u_{0}\right|_{M^{d(p-1) / \alpha}}>C(\alpha, d, p)$ implies that each nonnegative solution of problem (6) with the initial condition (2) blows up in a finite time.

It is of interest to estimate the discrepancy of these constants $c(\alpha, d, p)$ and $C(\alpha, d, p)$ compared to the Morrey space norm

$$
\left|u_{\infty}\right|_{M_{q}^{d(p-1) / \alpha}}=\left(\frac{\sigma_{d}}{d-\frac{\alpha}{p-1}}\right)^{1 / q} s(\alpha, d, p)
$$

of the singular stationary solution $u_{\infty}>0$ of (6) which exists for $p>1+\frac{\alpha}{d-\alpha}$; here

$$
\sigma_{d}=\frac{2 \pi^{d / 2}}{\Gamma\left(\frac{d}{2}\right)}
$$

is the area of the unit sphere $\mathbb{S}^{d-1}$ in $\mathbb{R}^{d}$. This singular stationary solution $u_{\infty}>0$ is homogeneous, see [8, Prop. 2.1],

$$
u_{\infty}(x)=s(\alpha, d, p)|x|^{-\frac{\alpha}{p-1}}
$$

with the constant

$$
s(\alpha, d, p)=\left(\frac{2^{\alpha}}{\Gamma\left(\frac{\alpha}{2(p-1)}\right)} \frac{\Gamma\left(\frac{d}{2}-\frac{\alpha}{2(p-1)}\right) \Gamma\left(\frac{p \alpha}{2(p-1)}\right)}{\Gamma\left(\frac{d}{2}-\frac{p \alpha}{2(p-1)}\right)}\right)^{\frac{1}{p-1}}
$$

Note that asymptotically

$$
s(\alpha, d, p) \approx c_{\alpha, p} d^{\frac{\alpha}{2(p-1)}} \quad \text { as } \quad d \rightarrow \infty
$$

with constants $c_{\alpha, p}$ independent of $d$. 
Of course, there are many interesting behaviors of solutions (and still open questions) for the initial data of intermediate size satisfying

$$
c(\alpha, d, p) \leq\left|u_{0}\right|_{M^{d(p-1) / \alpha}} \leq C(\alpha, d, p),
$$

and/or suitable pointwise estimates comparing the initial condition $u_{0}$ with the singular solution $u_{\infty}$, see e.g., [8]. One of the results in this direction is [8, Theorem 2.6]: if $\alpha \in(0,2), p>1+\frac{\alpha}{d-\alpha}\left(>1+\frac{\alpha}{d}\right), u=u(x, t)$ is a solution of problem (6) with (2) and $0 \leq u_{0}(x) \leq u_{\infty}(x)$ (plus some qualitative assumptions like $u_{0} \in M^{d(p-1) / \alpha}\left(\mathbb{R}^{d}\right) \cap$ $M^{\tilde{s}}\left(\mathbb{R}^{d}\right)$ with $\tilde{s}>d(p-1) / \alpha, \lim _{x \rightarrow 0}|x|^{\frac{\alpha}{p-1}} u(x, t)=\lim _{x \rightarrow \infty}|x|^{\frac{\alpha}{p-1}} u(x, t)=0$, uniformly in $t \in(0, T))$, then $u$ can be continued to a global in time solution which still satisfies the bound $0 \leq u(x, t) \leq u_{\infty}(x)$.

These are natural extensions of properties of the Cauchy problem (5) with (2) studied in, e.g., [4,17,18].

Once again, here it should be stressed on the fact that conditions on initial data guaranteeing local-in-time existence of solutions of Eq. (5) derived in [2] but motivated by [3], and then interpreted in [4] as a bound on the Morrey space $M^{d(p-1) / 2}\left(\mathbb{R}^{d}\right)$ norm, are qualitatively close to those above even if they involve $M_{q}^{s}\left(\mathbb{R}^{d}\right)$ spaces with any $q>1$, see also [4, Remark 3.4], [18, Proposition 6.1].

\section{Estimates of discrepancy}

Similarly to the considerations in [9] on blowup for radial solutions of chemotaxis systems, we determine asymptotic (with respect to the variable of dimension $d \rightarrow \infty$ ) discrepancy between bounds in sufficient conditions for blowup either in terms of multiple of the singular solution or in terms of critical value of the radial concentration (and therefore of the Morrey norm) of the initial data for the model problem (6) with data (2).

Theorem 5 (i) For each $\alpha \in(0,2]$ and $p>1+\frac{\alpha}{d}$ there exists a constant $v_{\alpha, p}$ independent of the dimension $d$ such that if $N>v_{\alpha, p}$, then each solution of the Cauchy problem (6) with data (2) in $\mathbb{R}^{d}$ with the initial data $u_{0}(x) \geq N u_{\infty}(x)$ blows up in a finite time.

(ii) For $\alpha=2$ and $p>1+\frac{2}{d}$ there exists a constant $\kappa_{2, p}$ independent of $d$ such that if the $\frac{d(p-1)}{2}$-radial concentration of $u_{0} \geq 0$ defined by

$$
\left\|u_{0}\right\|_{\frac{d(p-1)}{2}} \equiv \sup _{r>0} r^{\frac{2}{p-1}-d} \int_{\{|y|<r\}} u_{0}(y) \mathrm{d} y
$$

satisfies

$$
\left\|u_{0}\right\|_{\frac{d(p-1)}{2}} \geq \kappa \sigma_{d} d^{1 / 2(p-1)}
$$

with some $\kappa>\kappa_{2, p}$ then each solution of (6) with (2) in $\mathbb{R}^{d}$ blows up in a finite time.

(iii) For $\alpha \in(0,2)$ and $p>1+\frac{\alpha}{d}\left(p>1+\frac{\alpha}{d-2}\right.$ so that $\left.d \geq 3\right)$ there exists a constant $\kappa_{\alpha, p}$ independent of $d$ such that if the $\frac{d(p-1)}{\alpha}$-radial concentration of $u_{0} \geq 0$ defined by 


$$
\left\|u_{0}\right\|_{\frac{d(p-1)}{\alpha}} \equiv \sup _{r>0} r^{\frac{\alpha}{p-1}-d} \int_{\{|y|<r\}} u_{0}(y) \mathrm{d} y
$$

satisfies

$$
\left\|u_{0}\right\|_{\frac{d(p-1)}{\alpha}} \geq \kappa \sigma_{d} d^{\alpha / 2(p-1)}
$$

with $\kappa>\kappa_{\alpha, p}$ then each solution of (6) with (2) blows up in a finite time.

Remark 6 The $\frac{d(p-1)}{\alpha}$-radial concentration defined in (32) is comparable with the Morrey norm in $M^{d(p-1) / \alpha}\left(\mathbb{R}^{d}\right)$

$$
c_{d}\left|u_{0}\right|_{M^{d(p-1) / \alpha}} \leq \sup _{r>0} r^{\frac{\alpha}{p-1}-d} \int_{\{|y|<r\}} u_{0}(y) \mathrm{d} y \leq\left|u_{0}\right|_{M^{d(p-1) / \alpha}} .
$$

However, the comparison constant $c_{d}$ depends heavily on $d$, cf. [6, Proposition 7.1]). Proof A more detailed analysis of condition (21) reveals that

$$
\sup _{t>0} t^{\frac{1}{p-1}} \mathrm{e}^{-t(-\Delta)^{\alpha / 2}} u_{0}(0)>c_{\alpha, p}
$$

is a sufficient condition for blowup, with some constant $c_{\alpha, p}>0$ independent of $d$. For $\alpha=2$ we simply have $c_{2, p}=\left(\frac{1}{p-1}\right)^{\frac{1}{p-1}}$, see [4].

First, we compute

$$
\begin{aligned}
K_{2, p}(d) & \equiv \sup _{t>0} t^{\frac{1}{p-1}} \mathrm{e}^{t \Delta}\left(u_{\infty}\right)(0) \\
& =s(2, d, p) \sup _{t>0} t^{\frac{1}{p-1}-\frac{d}{2}} \sigma_{d} \int_{0}^{\infty} \mathrm{e}^{-r^{2} / 4 t}(4 \pi)^{-d / 2} r^{-\frac{2}{p-1}+d-1} \mathrm{~d} r \\
& =s(2, d, p) 4^{-\frac{1}{p-1}} \frac{2}{\Gamma\left(\frac{d}{2}\right)} \int_{0}^{\infty} \mathrm{e}^{-\tau} \tau^{\frac{d-1}{2}-\frac{1}{p-1}-\frac{1}{2}} \mathrm{~d} \tau \\
& =s(2, d, p) 2^{1-\frac{2}{p-1}} \frac{\Gamma\left(\frac{d}{2}-\frac{1}{p-1}\right)}{\Gamma\left(\frac{d}{2}\right)} \\
& \asymp d^{\frac{1}{p-1}-\frac{1}{p-1}} \asymp 1 .
\end{aligned}
$$

The last two lines follow using the relation

$$
\frac{\Gamma(z+a)}{\Gamma(z+b)} \asymp z^{a-b} \text { as } z \rightarrow \infty
$$

see e.g. [21], which is an immediate consequence of the Stirling formula

$$
\Gamma(z+1) \approx \sqrt{2 \pi z} z^{z} \mathrm{e}^{-z} \text { as } z \rightarrow \infty
$$


Next, for $\alpha \in(0,2)$ we need a dimension-free representation of the kernel of the semigroup $\mathrm{e}^{-t(-\Delta)^{\alpha / 2}}$ using the Bochner subordination formula, cf. [22, Ch. IX.11]

$$
\mathrm{e}^{-t(-\Delta)^{\alpha / 2}}=\int_{0}^{\infty} f_{t, \alpha}(\lambda) \mathrm{e}^{\lambda \Delta} \mathrm{d} \lambda, \quad \text { or } P_{t, \alpha}(x)=\int_{0}^{\infty} f_{t, \alpha}(\lambda) P_{t, 2}(x) \mathrm{d} \lambda,
$$

with some functions $f_{t, \alpha}(\lambda) \geq 0$ independent of $d$. In fact, the subordinators $f_{t, \alpha}$ satisfy $\mathrm{e}^{-t a^{\alpha}}=\int_{0}^{\infty} f_{t, \alpha}(\lambda) \mathrm{e}^{-\lambda a} \mathrm{~d} \lambda$, so that they have selfsimilar form $f_{t, \alpha}(\lambda)=$ $t^{-\frac{1}{\alpha}} f_{1, \alpha}\left(\lambda t^{-\frac{1}{\alpha}}\right)$.

Then, we have extensions of the previous computations

$$
\begin{aligned}
K_{\alpha, p}(d)= & \sup _{t>0} t^{\frac{1}{p-1}} \mathrm{e}^{-t(-\Delta)^{\alpha / 2}}\left(\frac{s(\alpha, d, p)}{|x|^{\frac{a}{p-1}}}\right)(0) \\
= & s(\alpha, d, p) \sup _{t>0} t^{\frac{1}{p-1}-\frac{d}{\alpha}} \sigma_{d} \int_{0}^{\infty} R\left(\frac{r}{t^{1 / \alpha}}\right) r^{-\frac{\alpha}{p-1}+d-1} \mathrm{~d} r \\
= & s(\alpha, d, p) \sigma_{d} \int_{0}^{\infty} R(\varrho) \varrho^{d-1-\frac{\alpha}{p-1}} \mathrm{~d} \varrho \\
= & s(\alpha, d, p) \sigma_{d} \int_{0}^{\infty} \int_{0}^{\infty} f_{1, \alpha}(\lambda)(4 \pi)^{-d / 2} \lambda^{-d / 2} \mathrm{e}^{-\varrho^{2} / 4 \lambda} \varrho^{d-1-\frac{\alpha}{p-1}} \mathrm{~d} \lambda \mathrm{d} \varrho \\
\approx & s(\alpha, d, p) \frac{1}{\Gamma\left(\frac{d}{2}\right)} 4^{-\frac{\alpha}{2(p-1)}} \int_{0}^{\infty} f_{1, \alpha}(\lambda) \lambda^{-\frac{\alpha}{2(p-1)}} \mathrm{d} \lambda \\
& \times \int_{0}^{\infty} \mathrm{e}^{-\tau} \tau^{\frac{d}{2}-1-\frac{\alpha}{2(p-1)}} \mathrm{d} \tau \asymp s(\alpha, d, p) \frac{\Gamma\left(\frac{d-\frac{\alpha}{p-1}}{2}\right)}{\Gamma\left(\frac{d}{2}\right)} \\
\asymp & d^{\frac{\alpha}{2(p-1)}-\frac{\alpha}{2(p-1)} \asymp 1}
\end{aligned}
$$

by representation (39), relations (29) and (37).

Using relations (36), (40) and the comparison principle for Eq. (6), we see that

$$
N \kappa_{\alpha, p}(d)>c_{\alpha, p}
$$

suffices to a finite time blowup, thus (i) follows since the bound for $K_{\alpha, p}(d)$ is $d$-independent.

(ii) Now, let us compute the asymptotics of the expression in condition (21) for $\alpha=2$ and the normalized Lebesgue measure $\mathrm{d} S$ on the unit sphere $\mathbb{S}^{d-1}$

$$
\begin{aligned}
L_{2, p}(d) & =\sup _{t>0} t^{\frac{1}{p-1}} \mathrm{e}^{-t \Delta}\left(\frac{\mathrm{d} S}{\sigma_{d}}\right) \\
& =\sup _{t>0}(4 \pi)^{-d / 2} t^{\frac{1}{p-1}-\frac{d}{2}} \mathrm{e}^{-1 / 4 t} \\
& =4^{-\frac{1}{p-1}} \pi^{-d / 2}\left(\frac{1}{\mathrm{e}}\left(\frac{d}{2}-\frac{1}{p-1}\right)\right)^{\frac{d}{2}-\frac{1}{p-1}}
\end{aligned}
$$




$$
\begin{aligned}
& \approx 4^{-\frac{1}{p-1}} \frac{1}{\sigma_{d}} \frac{1}{\Gamma\left(\frac{d}{2}\right)} \frac{\Gamma\left(\frac{d}{2}-\frac{1}{p-1}+1\right)}{\left(2 \pi\left(\frac{d}{2}-\frac{1}{p-1}\right)\right)^{1 / 2}} \\
& \asymp \frac{1}{\sigma_{d}} d^{\frac{1}{2}-\frac{1}{p-1}} .
\end{aligned}
$$

Indeed, for $\frac{d}{2}>\frac{1}{p-1}$ the quantity $\max _{t>0} t^{\frac{1}{p-1}-\frac{d}{2}} \mathrm{e}^{-1 / 4 t}$ is attained for $t_{0}=$ $\left(4\left(\frac{d}{2}-\frac{1}{p-1}\right)\right)^{-1}$, and then relation (37) is used.

(iii) For $\alpha \in(0,2)$, instead of (31) an analogous sufficient condition is of different order than for $\alpha=2$, namely the following

$$
\left\|u_{0}\right\|_{\frac{d(p-1)}{\alpha}}=\sup _{r>0} r^{\frac{\alpha}{p-1}-d} \int_{\{|y|<r\}} u_{0}(y) \mathrm{d} y \geq \kappa_{\alpha, p} \sigma_{d} d^{\alpha / 2(p-1)}
$$

with a constant $\kappa_{\alpha, p}$ independent of $d$. Indeed, for the normalized Lebesgue measure $\mathrm{d} S$ on the unit sphere $\mathbb{S}^{d-1}$ we have an upper bound for the quantity

$$
\begin{aligned}
L_{\alpha, p}(d) & =\sup _{t>0} t^{\frac{1}{p-1}} \mathrm{e}^{-t(-\Delta)^{\alpha / 2}}\left(\frac{\mathrm{d} S}{\sigma_{d}}\right) \\
& =\sup _{t>0} t^{\frac{1}{p-1}-\frac{d}{\alpha}} R\left(\frac{1}{t^{1 / \alpha}}\right) \\
& =\sup _{\varrho>0} \varrho^{d-\frac{\alpha}{p-1}} R(\varrho) \\
& =\sup _{\varrho>0} \int_{0}^{\infty} f_{1, \alpha}(\lambda)(4 \pi \lambda)^{-d / 2} \varrho^{d-\frac{\alpha}{p-1}} \mathrm{e}^{-\varrho^{2} / 4 \lambda} \mathrm{d} \lambda \\
& \leq 4^{-\frac{\alpha}{2(p-1)}} \frac{2}{\sigma_{d} \Gamma\left(\frac{d}{2}\right)} \int_{0}^{\infty} \sup _{\varrho>0}\left(f_{1, \alpha}\left(\frac{\varrho^{2}}{4 \tau}\right)\left(\frac{\varrho^{2}}{4 \tau}\right)^{1-\frac{\alpha}{2(p-1)}}\right) \tau^{\frac{d}{2}-\frac{\alpha}{2(p-1)}-1} \mathrm{e}^{-\tau} \mathrm{d} \tau \\
& \asymp \frac{1}{\sigma_{d} \Gamma\left(\frac{d}{2}\right)} \Gamma\left(\frac{d-\frac{a}{p-1}}{2}\right) \\
& \asymp \frac{1}{\sigma_{d}} d^{-\frac{\alpha}{2(p-1)}}
\end{aligned}
$$

since representation (39), formulas (26) and (37) hold.

For the derivation of an asymptotic lower bound on the quantity $L_{\alpha, p}(d)$, begin with the observation that for $\beta=\frac{d}{2}-\frac{\alpha}{2(p-1)}-1(\beta>0$ since $\left.p>1+\frac{\alpha}{d-2}\right)$ 


$$
\begin{aligned}
m \equiv \max _{\tau>0} \mathrm{e}^{-\tau} \tau^{\beta} & =\mathrm{e}^{-\tau_{0}} \tau_{0}^{\beta} \text { with } \tau_{0}=\beta \\
& =\mathrm{e}^{-\beta} \beta^{\beta} \\
& \approx \Gamma(\beta+1) \frac{1}{\sqrt{2 \pi \beta}}
\end{aligned}
$$

holds by (38). Now, let $h \asymp d^{\frac{1}{2}}$. It is easy to check that

$$
\frac{1}{m} \min _{\left[\tau_{0}, \tau_{0}+h\right]} \mathrm{e}^{-\tau} \tau^{\beta} \geq \eta
$$

for some $\eta>0$, uniformly in $d$. Indeed,

$$
\log \frac{(d+h)^{d} \mathrm{e}^{-d-h}}{d^{d} \mathrm{e}^{-d}}=d \log \left(1+\frac{h}{d}\right)-h \approx d \frac{h}{d}-\frac{d h^{2}}{2 d^{2}}-h=\mathcal{O}\left(\frac{h^{2}}{2 d}\right) .
$$

From formulas (43) and (44) we infer

$$
\begin{aligned}
L_{\alpha, p}(d) & \geq 4^{-\frac{\alpha}{2(p-1)}} \frac{2}{\sigma_{d} \Gamma\left(\frac{d}{2}\right)} \sup _{\varrho>0} \int_{\tau_{0}}^{\tau_{0}+h}\left(f_{1, \alpha}\left(\frac{\varrho^{2}}{4 \tau}\right)\left(\frac{\varrho^{2}}{4 \tau}\right)^{1-\frac{\alpha}{2(p-1)}}\right) \tau^{\frac{d}{2}-\frac{\alpha}{2(p-1)}-1} \mathrm{e}^{-\tau} \mathrm{d} \tau \\
& \geq 4^{-\frac{\alpha}{2(p-1)}} \frac{2}{\sigma_{d} \Gamma\left(\frac{d}{2}\right)} \frac{\eta h}{\sqrt{d}} \Gamma\left(\frac{d}{2}-\frac{\alpha}{2(p-1)}\right) \\
& \asymp \frac{1}{\sigma_{d}} d^{-\frac{\alpha}{2(p-1)}} .
\end{aligned}
$$

Therefore the relation $L_{\alpha, p}(d) \asymp \frac{1}{\sigma_{d}} d^{-\frac{\alpha}{2(p-1)}}$ holds. This is an estimate of optimal order and different from its counterpart for $\alpha=2$. Now, it is clear that a sufficient condition for blowup is satisfied if

$$
N L_{\alpha, p}(d)>c_{\alpha, p}
$$

with either $N=\kappa \sigma_{d} d^{1 /(p-1)}$ if $\alpha=2$ or $N=\kappa \sigma_{d} d^{\alpha / 2(p-1)}$ if $\alpha \in(0,2)$.

\section{Concluding remarks}

The classical idea of the proof of blowup of solutions by Fujita [12] has been applied in the wider context of problems with general linear diffusion operators and convex nonlinearities leading to qualitatively simple sufficient conditions for blowup in problems with nice approximative scaling properties.

For more classical problems involving diffusions defined by either the Laplacian or its fractional powers, these conditions have been compared with results guaranteeing the existence of global solutions. The discrepancies between bounds on quantities determining local/global behavior of solutions have been estimated. 
Open Access This article is distributed under the terms of the Creative Commons Attribution 4.0 International License (http://creativecommons.org/licenses/by/4.0/), which permits unrestricted use, distribution, and reproduction in any medium, provided you give appropriate credit to the original author(s) and the source, provide a link to the Creative Commons license, and indicate if changes were made.

\section{References}

1. Alfaro, M.: Fujita blow up phenomena and hair trigger effect: the role of dispersal tails. Ann. Inst. Henri Poincaré, Anal. Non Linéaire 34, 1309-1327 (2017)

2. Andreucci, D., DiBenedetto, E.: On the Cauchy problem and initial traces for a class of evolution equations with strongly nonlinear sources. Ann. Sc. Norm. Super. Pisa. Cl. Sci., IV. Ser. 18, 363-441 (1991)

3. Baras, P., Pierre, M.: Critère d'existence de solutions positives pour des équations semi-linéaires non monotones. Ann. Inst. Henri Poincaré, Anal. Non Linéaire 2, 185-212 (1985)

4. Biler, P.: Blowup versus global in time existence of solutions for nonlinear heat equations. Topol. Methods Nonlin. Analysis 52, 147-160 (2018)

5. Biler, P.: Singularities of Solutions to Chemotaxis Systems. Series in Mathematics and Life Sciences. De Gruyter (in preparation)

6. Biler, P., Karch, G., Zienkiewicz, J.: Large global-in-time solutions to a nonlocal model of chemotaxis. Adv. Math. 330, 834-875 (2018)

7. Biler, P., Karch, G., Pilarczyk, D.: Global radial solutions in classical Keller-Segel chemotaxis model, 1-20, submitted. arXiv:1807.02628

8. Biler, P., Pilarczyk, D.: Around a singular solution of a nonlocal nonlinear heat equation, 1-20, NoDEA, Nonlinear Differ. Equ. Appl. (2019). https://doi.org/10.1007/s00030-019-0552-z

9. Biler, P., Zienkiewicz, J.: Blowing up radial solutions in the minimal Keller-Segel chemotaxis model, 1-20. J. Evol. Equ. (2018). https://doi.org/10.1007/s00028-018-0469-8

10. Burczak, J., Granero-Belinchón, R.: Global solutions for a supercritical drift-diffusion equation. Adv. Math. 295, 334-367 (2016)

11. Chasseigne, E., Chaves, M., Rossi, J.D.: Asymptotic behavior for nonlocal diffusion equations. J. Math. pures appl. 86, 271-291 (2006)

12. Fujita, H.: On the blowing up of solutions of the Cauchy problem for $u_{t}=\Delta u+u^{1+\alpha}$. J. Fac. Sci. Univ. Tokyo Sect. I(13), 109-124 (1966)

13. García-Melián, J., Quirós, F.: Fujita exponents for evolution problems with nonlocal diffusion. J. Evol. Equ. 10, 147-161 (2010)

14. Laister, R., Robinson, J.C., Sierżęga, M., Vidal-López, A.: A complete characterisation of local existence for semilinear heat equations in Lebesgue spaces. Ann. Inst. Henri Poincaré, Anal. Non Linéaire 33, 1519-1538 (2016)

15. Lemarié-Rieusset, P.-G.: Small data in an optimal Banach space for the parabolic-parabolic and parabolic-elliptic Keller-Segel equations in the whole space. Adv. Diff. Eq. 18, 1189-1208 (2013)

16. Lemarié-Rieusset, P.-G.: Sobolev multipliers, maximal functions and parabolic equations with a quadratic nonlinearity. J. Funct. Anal. 274, 659-694 (2018)

17. Quittner, P., Souplet, P.: Superlinear parabolic problems. Blow-up, global existence and steady states. Birkhäuser Advanced Texts, Basel (2007)

18. Souplet, $\mathrm{Ph}$ : Morrey spaces and classification of global solutions for a supercritical semilinear heat equation in $\mathbb{R}^{n}$. J. Funct. Anal. 272, 2005-2037 (2017)

19. Souplet, $\mathrm{Ph}$, Weissler, F.B.: Regular self-similar solutions of the nonlinear heat equation with initial data above the singular steady state. Ann. Inst. Henri Poincaré, Anal. Non Linéaire 20, 213-235 (2003)

20. Sugitani, S.: On nonexistence of global solutions for some nonlinear integral equations. Osaka J. Math. 12, 45-51 (1975)

21. Tricomi, F.G., Erdélyi, A.: The asymptotic expansion of a ratio of Gamma functions. Pac. J. Math. 1, 133-142 (1951)

22. Yosida, K.: Functional Analysis, 6th edn. Springer, Berlin (1980)

Publisher's Note Springer Nature remains neutral with regard to jurisdictional claims in published maps and institutional affiliations. 